\title{
BLOOD OXYGEN STUDIES IN PATIENTS WITH POLYCYTHEMIA AND IN NORMAL SUBJECTS ${ }^{1}$
}

\author{
By L. R. WASSERMAN, ${ }^{2}$ R. L. DOBSON, AND J. H. LAWRENCE \\ (From the Division of Medical Physics and Division of Medicine, \\ University of California, Berkeley ${ }^{3}$ )
}

(Received for publication December 22, 1947)

During the past few years various studies have been undertaken in this laboratory in an attempt to elucidate the pathological physiology and nature of polycythemia vera. Although the etiology remains obscure, interesting data have been accumulated on (1) blood oxygen saturation, (2) $\mathrm{O}_{2}$ consumption and $\mathrm{CO}_{2}$ production, (3) diffusion of gases across the alveolus-blood barrier, and (4) bone marrow findings. The present paper is concerned with a brief discussion of blood oxygen saturation in polycythemia and the results of determinations made on 74 subjects, 48 of whom were suffering from polycythemia vera. Subsequent reports will cover the remaining phases of the investigations and others.

\section{METHODS}

The diagnosis of polycythemia vera was made by the usual clinical and hematologic methods which included a complete blood count, packed cell volume, and sternal marrow aspiration. Blood volumes were done on some patients employing the dye $\mathrm{T}-1824$, radioactive colloids of zirconium and yttrium (1), the $\mathrm{CO}$ method, or by the use of radioactively labeled red cells. Viscosity determinations and circulation times were also done in most cases. All patients had chest $\mathrm{X}$-rays to exclude pulmonary pathology, and no patient with a chronic cardiac or pulmonary history was included in this series except as noted in the cases of secondary polycythemia.

Blood was drawn from the resting patient anaerobically from either the radial or brachial artery, and the oxygen content and capacity determined by the micro gasometric technique of Roughton and Scholander (2). Arterial capillary blood from the lobe of the ear drawn anaerobically into a tuberculin syringe by means of a small glass ear cup was found to give the same results, and after sufficient controls had been done the ear method

1 This work has been supported first by a grant from the International Cancer Research Foundation and later by one from the U. S. Public Health Service.

2 Aided by a grant from the Sara Welt Fund; from the Physics Laboratory and Medical Services, Mt. Sinai Hospital, New York.

3 From the Donner Laboratory of Medical Physics and the Radiation Laboratorv. was used exclusively. In this method the whole ear is warmed for one-half hour by placing over it an anesthesia mask containing a small electric bulb. After warming, a small incision with a No. 11 surgical blade will produce a free flow of arterial capillary blood which can be rapidly withdrawn into the syringe containing a drop of mercury and previously rinsed with either heparin or a heparin-fluoride mixture. After the blood is collected, the ear cup is removed from the short rubber tubing connecting it to the syringe, and the tubing is turned on itself and made airtight with a rubber band. The drop of mercury is then used to mix the blood and anticoagulant by rapidly turning the syringe from side to side. The analysis must be made within a few hours, and the syringe with blood must be kept on ice at all times except when the actual determinations are being made.

\section{RESULTS}

For purposes of comparison the cases of polycythemia vera were divided into two groups, those with hematocrits below $50 \%$ and those above. A total of 48 cases of polycythemia vera was examined on whom 97 oxygen saturation determinations were done, 70 by ear capillary method and 27 by the usual radial or brachial artery method (Tables I and II). Analyses of the oxygen saturation were also done in 18 normal persons (Table III), in five patients with hypochromic anemia (Table IV) and in three cases of erythrocytosis secondary to chronic pulmonary or cardiac disease (Table V).

Eight cases of polycythemia vera had packed red cell volumes below $50 \%$. These patients had been treated with either phlebotomies or radioactive phosphorus before the oxygen determinations were done. The average hematocrit was $44.5 \pm 0.62$ and ranged from 41.5 to 46.8 . The blood oxygen saturation determined on ear capillary blood ranged from 90.0 to $98.4 \%$ with an average of $93.9 \pm 1.1 \%$. In four of these cases arterial blood from the brachial artery gave a slightly lower oxygen saturation, the results ranging from 88.2 to $93.8 \%$ with an average of $91.3 \pm$ $.3 \%$. 
BLOOD OXYGEN STUDIES IN POLYCYTHEMIA VERA

TABLE I

Results in polycythemia vera with hematocrits of $50 \%$ or over

\begin{tabular}{|c|c|c|c|c|c|c|c|c|c|c|c|c|c|}
\hline \multirow{2}{*}{ Case No. } & \multirow[b]{2}{*}{ Hgb. } & \multirow{2}{*}{$\underset{106 / \mathrm{mm} \cdot .^{2}}{\mathrm{RBC}}$} & \multirow{2}{*}{$\underset{\text { atocrit }}{\text { Hem- }}$} & \multirow{2}{*}{\multicolumn{2}{|c|}{ Blood Volume }} & \multicolumn{4}{|c|}{ Ear } & \multicolumn{4}{|c|}{ Artery } \\
\hline & & & & & & & & $\mathrm{O}_{2}$ & & & & $\mathrm{O}_{2}$ & \\
\hline $\begin{array}{l}1 \\
2 \\
3 \\
4 \\
5\end{array}$ & $\begin{array}{l}\text { gms. } \\
17.6 \\
24.3 \\
29.8 \\
17.9 \\
19.0\end{array}$ & $\begin{array}{l}7.17 \\
7.96 \\
8.1 \\
6.4 \\
6.3\end{array}$ & $\begin{array}{l}\% \\
60 \\
75 \\
76 \\
55.8 \\
57.5\end{array}$ & $\begin{array}{l}\text { liters } \\
R B C\end{array}$ & $\begin{array}{c}\text { liters } \\
\text { plasma }\end{array}$ & $\begin{array}{c}\text { vol. \% } \\
21.9 \\
28.8 \\
36.3 \\
22.1 \\
24.4\end{array}$ & $\begin{array}{l}\text { sol. \% } \\
22 \\
31 \\
37.8 \\
23.0 \\
24.8\end{array}$ & \begin{tabular}{l}
\multicolumn{1}{c}{$\%$} \\
95 \\
93 \\
96.2 \\
96 \\
98.5
\end{tabular} & $\begin{array}{c}\text { hrs. } \\
3 \\
4 \\
2 \\
1 \\
1\end{array}$ & $\begin{array}{l}\text { øol. \% } \\
27.3 \\
36.0 \\
22.3\end{array}$ & $\begin{array}{l}\text { ool. \% } \\
30.4 \\
37.6 \\
23.0\end{array}$ & $\begin{array}{l}\% \\
90 \\
95.8 \\
97 .\end{array}$ & $\begin{array}{l}\text { hrs. } \\
5 \\
3 \\
2\end{array}$ \\
\hline $\begin{array}{r}6 \\
7 \\
8 \\
9 \\
10\end{array}$ & $\begin{array}{l}20.7 \\
18.5 \\
20.1 \\
25.3 \\
22.5\end{array}$ & $\begin{array}{l}7.96 \\
6.03 \\
7.07 \\
9.84 \\
9.22\end{array}$ & $\begin{array}{l}70 \\
55 \\
60.6 \\
81 \\
79\end{array}$ & $\begin{array}{c}3.7 \\
4.4 \\
9.0 \pm 2.0 \\
-\end{array}$ & $\begin{array}{c}3.0 \\
2.8 \\
2.0 \pm 0.4 \\
-\end{array}$ & $\begin{array}{l}25.0 \\
23.7 \\
25.6 \\
30.4 \\
25.7\end{array}$ & $\begin{array}{l}26.6 \\
24.1 \\
26.6 \\
32.8 \\
27.6\end{array}$ & $\begin{array}{l}94 \\
98.4 \\
96.2 \\
92.5 \\
93.0\end{array}$ & $\begin{array}{l}2 \\
1 \\
1 \\
4 \\
2\end{array}$ & $\begin{array}{l}23.9 \\
30.5\end{array}$ & $\begin{array}{l}24.3 \\
33.2\end{array}$ & $\begin{array}{l}98.4 \\
92.0\end{array}$ & $\begin{array}{l}2 \\
4.5\end{array}$ \\
\hline $\begin{array}{l}11 \\
12 \\
13 \\
14 \\
15\end{array}$ & $\begin{array}{l}17.3 \\
18.9 \\
18.9 \\
17.0 \\
15.2\end{array}$ & $\begin{array}{l}9.24 \\
5.32 \\
5.96 \\
6.18 \\
6.82\end{array}$ & $\begin{array}{l}62 \\
54 \\
56 \\
60 \\
50\end{array}$ & $\begin{array}{l}4.4 \\
3.8 \\
4.2 \\
3.2\end{array}$ & $\begin{array}{l}2.7 \\
3.3 \\
3.5 \\
\\
3.2\end{array}$ & $\begin{array}{l}20.0 \\
22.8 \\
23.3 \\
20.2 \\
18.9\end{array}$ & $\begin{array}{l}22.0 \\
24.1 \\
24.1 \\
22.2 \\
20.0\end{array}$ & $\begin{array}{l}91 \\
95 \\
96.8 \\
91.0 \\
92.4\end{array}$ & $\begin{array}{l}3 \\
4 \\
2 \\
6 \\
5\end{array}$ & 20.2 & 22.2 & 91.0 & 4 \\
\hline $\begin{array}{l}16 \\
17 \\
18 \\
19 \\
20\end{array}$ & $\begin{array}{l}23.4 \\
15.8 \\
22.5 \\
18.5 \\
22.9\end{array}$ & $\begin{array}{r}7.82 \\
8.66 \\
7.76 \\
6.24 \\
10.82\end{array}$ & $\begin{array}{l}63 \\
70 \\
69 \\
52 \\
70\end{array}$ & & & $\begin{array}{l}28.7 \\
20.3 \\
28.3 \\
22.0 \\
27.0\end{array}$ & $\begin{array}{l}29.9 \\
20.6 \\
29.6 \\
24.1 \\
29.7\end{array}$ & $\begin{array}{l}96.1 \\
98.5 \\
95.5 \\
91.4 \\
92.5\end{array}$ & $\begin{array}{l}4 \\
3 \\
3 \\
4 \\
4\end{array}$ & & & & \\
\hline $\begin{array}{l}21 \\
22 \\
23 \\
24 \\
25\end{array}$ & $\begin{array}{l}23.1 \\
17.3 \\
18.5 \\
19.2 \\
17.3\end{array}$ & $\begin{array}{l}7.62 \\
5.78 \\
7.56 \\
7.78 \\
7.78\end{array}$ & $\begin{array}{l}66 \\
50.5 \\
63 \\
70 \\
55\end{array}$ & $\begin{array}{l}3.9 \\
3.2 \\
4.5\end{array}$ & $\begin{array}{l}2.6 \\
3.1 \\
2.5\end{array}$ & $\begin{array}{l}27.6 \\
21.7 \\
21.8 \\
23.7 \\
21.3\end{array}$ & $\begin{array}{l}30.0 \\
22.2 \\
24.0 \\
24.8 \\
22.1\end{array}$ & $\begin{array}{l}92 \\
97.5 \\
91.0 \\
95.5 \\
96.5\end{array}$ & $\begin{array}{l}3 \\
2 \\
3 \\
2 \\
2\end{array}$ & 28.1 & 30.0 & 93.8 & 4 \\
\hline $\begin{array}{l}26 \\
27 \\
28 \\
29 \\
30\end{array}$ & $\begin{array}{l}18 \\
23.5 \\
19.1 \\
23.5 \\
18\end{array}$ & $\begin{array}{c}6.9 \\
9.04 \\
10.2 \\
9.2 \\
7.09\end{array}$ & $\begin{array}{l}62.5 \\
72.3 \\
75.0 \\
77 \\
63\end{array}$ & & & $\begin{array}{l}22.2 \\
28.9 \\
23.0 \\
28.7 \\
22.8\end{array}$ & $\begin{array}{l}23.0 \\
31.1 \\
24.6 \\
30.8 \\
23.2\end{array}$ & $\begin{array}{l}96.5 \\
93.0 \\
93.3 \\
93.0 \\
98.0\end{array}$ & $\begin{array}{l}2 \\
2 \\
2 \\
1 \\
1\end{array}$ & & & & \\
\hline $\begin{array}{l}31 \\
32 \\
33 \\
34 \\
35\end{array}$ & $\begin{array}{l}15.9 \\
19.3 \\
16.5 \\
15.2 \\
19.1\end{array}$ & $\begin{array}{l}6.24 \\
9.85 \\
9.62 \\
6.7 \\
6.5\end{array}$ & $\begin{array}{l}51 \\
73 \\
60 \\
52 \\
58\end{array}$ & 4.3 & 3.4 & $\begin{array}{l}19.5 \\
22.7 \\
20.4 \\
19.2 \\
23.9\end{array}$ & $\begin{array}{l}20.3 \\
24.8 \\
21.4 \\
20 \\
25.1\end{array}$ & $\begin{array}{l}96.0 \\
91.5 \\
95.4 \\
96 \\
95\end{array}$ & $\begin{array}{l}1 \\
4 \\
3 \\
3 \\
1\end{array}$ & 22.9 & 24.4 & 94.0 & 1.5 \\
\hline $\begin{array}{l}36 \\
37 \\
38 \\
39 \\
40\end{array}$ & $\begin{array}{l}19.5 \\
19.2 \\
20.5 \\
22.0 \\
19.2\end{array}$ & $\begin{array}{l}7.5 \\
6.3 \\
9.2 \\
7.3 \\
6.5\end{array}$ & $\begin{array}{l}61 \\
57 \\
70 \\
97 \\
59\end{array}$ & $\begin{array}{l}4.6 \\
4.2 \\
5.4 \\
4.9 \\
4.2\end{array}$ & $\begin{array}{l}2.8 \\
3.1 \\
2.6 \\
2.3 \\
2.9\end{array}$ & $\begin{array}{l}23.3 \\
23.8 \\
25.6 \\
27.7 \\
24.0\end{array}$ & $\begin{array}{l}25.3 \\
25.0 \\
26.7 \\
28.9 \\
24.9\end{array}$ & $\begin{array}{l}92 \\
95 \\
96 \\
96 \\
96.5\end{array}$ & $\begin{array}{l}2 \\
1 \\
1 \\
1 \\
1\end{array}$ & $\begin{array}{l}20.0 \\
22.0 \\
26.0 \\
27.6 \\
23.8\end{array}$ & $\begin{array}{l}21.2 \\
23.0 \\
26.9 \\
28.7 \\
25.0\end{array}$ & $\begin{array}{l}94.0 \\
95.5 \\
96.5 \\
96.0 \\
95.0\end{array}$ & $\begin{array}{l}2.5 \\
2 \\
2 \\
2 \\
2\end{array}$ \\
\hline Average & & & 63.2 & & & & & 94.7 & & & & 94.5 & \\
\hline
\end{tabular}

The remaining 40 cases of erythremia had an average hematocrit of $63.2 \pm 1.8 \%$ with a mean oxygen saturation of $94.7 \pm 0.36 \%$ as determined on ear capillary blood. In 13 of these cases with an average packed cell volume of $67.3 \pm 3.9 \%$, the large artery blood oxygen saturation was $94.5 \pm$ $1.3 \%$.
Normal and hypochromic anemia cases examined in the same manner yielded similar results, viz., $95.1 \pm 0.59 \%$ and $96.0 \pm 1.1 \%$, respectively.

The three cases of secondary polycythemia yielded low oxygen saturation, the results ranging from 75 to $88 \%$ with a mean of $79.0 \pm 4.5 \%$. 
TABLE II

Polycythemia vera with hematocrits less than $50 \%$

\begin{tabular}{|c|c|c|c|c|c|c|c|c|c|c|c|c|c|}
\hline \multirow[b]{2}{*}{ Case No. } & \multirow[b]{2}{*}{ Hgb. } & \multirow{2}{*}{$\begin{array}{c}\underset{\mathrm{RBC}}{\times} \\
106 / \mathrm{mm}^{3} .\end{array}$} & \multirow[b]{2}{*}{$\underset{\text { crit }}{\text { Hemato- }}$} & & & \multicolumn{4}{|c|}{ Ear } & \multicolumn{4}{|c|}{ Artery } \\
\hline & & & & \multicolumn{2}{|c|}{ Blood Volume } & $\mathrm{O}_{3}$ & $U_{3}$ & $\mathrm{O}_{2}$ & Time & & & $\mathrm{O}_{2}$ & \\
\hline $\begin{array}{l}1 \\
2 \\
3 \\
4\end{array}$ & $\begin{array}{l}\text { gms. } \\
15.9 \\
13.9 \\
17.5 \\
18.0\end{array}$ & $\begin{array}{l}7.34 \\
5.48 \\
5.8 \\
5.62\end{array}$ & $\begin{array}{c}\% \\
46.8 \\
45.5 \\
42.9 \\
46.0\end{array}$ & $\begin{array}{c}\text { liters } \\
R B C \\
2.8\end{array}$ & $\begin{array}{c}\text { liters } \\
\text { plasma } \\
3.2 \\
\\
3.6\end{array}$ & $\begin{array}{c}\text { vol. } \% \\
18.9 \\
16.4 \\
22.6 \\
22.0\end{array}$ & $\begin{array}{c}\text { vol. } \% \\
21.0 \\
18.2 \\
23.0 \\
23.5\end{array}$ & $\begin{array}{c}\% \\
90.0 \\
90.0 \\
98.4 \\
93.5\end{array}$ & $\begin{array}{c}\text { hrs. } \\
4 \\
4 \\
2 \\
2\end{array}$ & $\begin{array}{c}\text { vol. \% } \\
18.5 \\
16.4\end{array}$ & $\begin{array}{c}\text { vol. \% } \\
21.0 \\
18.2\end{array}$ & $\begin{array}{c}\% \\
88.2 \\
90.0\end{array}$ & $\begin{array}{c}\text { hrs. } \\
5 \\
5\end{array}$ \\
\hline $\begin{array}{l}5 \\
6 \\
7 \\
8\end{array}$ & $\begin{array}{l}14.9 \\
16 \\
13.4 \\
14.9\end{array}$ & $\begin{array}{l}5.42 \\
5.56 \\
4.93 \\
6.66\end{array}$ & $\begin{array}{l}41.5 \\
46.0 \\
43.0 \\
45.0\end{array}$ & & & $\begin{array}{l}18.6 \\
19.8 \\
16.3 \\
18.8\end{array}$ & $\begin{array}{l}19.5 \\
20.6 \\
17.5 \\
19.6\end{array}$ & $\begin{array}{l}93.8 \\
96.0 \\
93.0 \\
96.3\end{array}$ & $\begin{array}{l}3 \\
1 \\
3 \\
2\end{array}$ & $\begin{array}{l}18.7 \\
16.4\end{array}$ & $\begin{array}{l}19.6 \\
17.5\end{array}$ & $\begin{array}{l}93.8 \\
93.5\end{array}$ & 4 \\
\hline Average & & & 44.5 & & & & & 93.9 & & & & 91.3 & \\
\hline
\end{tabular}

\section{DISCUSSION}

Few complete studies of arterial oxygen saturation in polycythemia vera are available. There is general agreement, however, among the scattered reports that saturation is within normal limits. In a total of 14 reported determinations in uncomplicated polycythemia vera results ranged from $91.0 \%$ to $97.0 \%$ (3-7), the average of $93.6 \%$ being slightly lower than an average of $95.5 \%$ reported for a series of 15 normal individuals (8). These figures compare favorably with those obtained in our study.

Bauer, Lawrowsky, and Skujin suggest after studying a large series of patients, two of whom had polycythemia vera, that there is a lessened degree of oxygen saturation of the hemoglobin as the amount of hemoglobin increases, so that the amount of oxygen carried by a given amount of

TABLE III

Results in normal subjects

\begin{tabular}{|c|c|c|c|c|c|c|c|c|c|c|c|}
\hline \multirow{2}{*}{ Case No. } & \multirow{2}{*}{ Hgb. } & \multirow{2}{*}{$\underset{106 / \mathrm{mm}^{2} .}{\mathrm{RBC}}$} & \multirow{2}{*}{$\underset{\text { crit }}{\text { Hemato- }}$} & \multicolumn{4}{|c|}{ Ear } & \multicolumn{4}{|c|}{ Artery } \\
\hline & & & & $\begin{array}{c}\mathrm{O}_{2} \\
\text { Content }\end{array}$ & $\begin{array}{c}\mathrm{O}_{2} \\
\text { Capacity }\end{array}$ & $\begin{array}{c}\mathrm{O}_{2} \\
\text { Saturation }\end{array}$ & Time & $\begin{array}{c}\mathrm{O}_{2} \\
\text { Content }\end{array}$ & $\begin{array}{c}\mathrm{O}_{2} \\
\text { Capacity }\end{array}$ & $\begin{array}{c}\mathrm{O}_{2} \\
\text { Saturation }\end{array}$ & Time \\
\hline $\begin{array}{l}1 \\
2 \\
3 \\
4 \\
5\end{array}$ & $\begin{array}{l}g m s . \\
15.6 \\
15.8 \\
12.9 \\
15.6 \\
15.3\end{array}$ & $\begin{array}{l}5.1 \\
5.2 \\
4.4 \\
5.3 \\
4.95\end{array}$ & $\begin{array}{l}\% \\
46 \\
46 \\
42 \\
47.8 \\
44.0\end{array}$ & $\begin{array}{c}\text { vol. \% } \\
20.1 \\
20.7 \\
16.8 \\
18.6 \\
18.7\end{array}$ & $\begin{array}{c}\text { vol. } \% \\
20.5 \\
20.8 \\
17.0 \\
20.3 \\
19.9\end{array}$ & \begin{tabular}{l}
\multicolumn{1}{c}{$\%$} \\
98.2 \\
99.4 \\
98.9 \\
90 \\
94.7
\end{tabular} & $\begin{array}{l}\text { hrs. } \\
1 \\
3 \\
1 \\
2 \\
1\end{array}$ & vol. \% & vol. \% & 94.0 & hrs. \\
\hline $\begin{array}{r}6 \\
7 \\
8 \\
9 \\
10\end{array}$ & $\begin{array}{l}15.8 \\
15.5 \\
14.3 \\
13.4 \\
17.0\end{array}$ & $\begin{array}{l}5.4 \\
5.2 \\
4.65 \\
4.45 \\
5.6\end{array}$ & $\begin{array}{l}48.0 \\
46.8 \\
42.4 \\
43 \\
48.4\end{array}$ & $\begin{array}{l}19.4 \\
18.6 \\
17.1 \\
16.7 \\
20.8\end{array}$ & $\begin{array}{l}20.8 \\
19.0 \\
17.9 \\
17.8 \\
22.5\end{array}$ & $\begin{array}{l}93.3 \\
98.0 \\
95.8 \\
93 \\
92\end{array}$ & $\begin{array}{l}1 \\
1.5 \\
2 \\
2 \\
3\end{array}$ & & & . & \\
\hline $\begin{array}{l}11 \\
12 \\
13 \\
14 \\
15\end{array}$ & $\begin{array}{l}15.1 \\
15.4 \\
15.5 \\
14.3 \\
13.6\end{array}$ & $\begin{array}{l}4.9 \\
4.7 \\
4.5 \\
4.7 \\
4.4\end{array}$ & $\begin{array}{l}44 \\
42.5 \\
42 \\
42 \\
43\end{array}$ & $\begin{array}{l}19.3 \\
20.8 \\
16.9 \\
18.4 \\
16.7\end{array}$ & $\begin{array}{l}20.2 \\
23.1 \\
17.7 \\
18.9 \\
17.7\end{array}$ & $\begin{array}{l}95 \\
90 \\
95 \\
97 \\
94\end{array}$ & $\begin{array}{l}2 \\
1 \\
2 \\
2 \\
3\end{array}$ & & & & \\
\hline $\begin{array}{l}16 \\
17 \\
18\end{array}$ & $\begin{array}{l}16.0 \\
15.8 \\
15.3\end{array}$ & $\begin{array}{l}5.2 \\
5.25 \\
5.01\end{array}$ & $\begin{array}{l}46 \\
47 \\
45\end{array}$ & $\begin{array}{l}19.7 \\
19.4 \\
20.0\end{array}$ & $\begin{array}{l}21.2 \\
21.1 \\
20.3\end{array}$ & $\begin{array}{l}93 \\
92 \\
98.5\end{array}$ & $\begin{array}{l}3 \\
3 \\
1\end{array}$ & & & & \\
\hline Average & & & 45.7 & & & 95.1 & & & & & \\
\hline
\end{tabular}


TABLE IV

Results of oxygen saturation in anemia

\begin{tabular}{|c|c|c|c|c|c|c|c|}
\hline \multirow{2}{*}{$\begin{array}{l}\text { Case } \\
\text { No. }\end{array}$} & \multirow{2}{*}{ Hgb. } & \multirow{2}{*}{$\begin{array}{c}\text { RBC } \\
\underset{\times}{\times} \\
10 \% / \mathrm{mm} \cdot .^{2}\end{array}$} & \multirow{2}{*}{$\underset{\text { crit }}{\text { Hemato- }}$} & \multicolumn{4}{|c|}{ Ear } \\
\hline & & & & $\begin{array}{c}\mathrm{O}_{2} \\
\text { Con- } \\
\text { tent }\end{array}$ & $\underset{\substack{\mathrm{O}_{2} \\
\text { ity }}}{\mathrm{Capac}^{-}}$ & $\begin{array}{c}\mathrm{O}_{2} \\
\text { Satu- } \\
\text { ration }\end{array}$ & Time \\
\hline $\begin{array}{l}1 \\
2 \\
3 \\
4 \\
5\end{array}$ & $\begin{array}{r}\text { gms. } \\
11.3 \\
11.7 \\
9.6 \\
12.5 \\
11.0\end{array}$ & $\begin{array}{l}4.05 \\
4.5 \\
3.6 \\
4.45 \\
4.2\end{array}$ & \begin{tabular}{l}
\multicolumn{1}{c}{$\%$} \\
33.9 \\
39.2 \\
26.3 \\
37 \\
35.0
\end{tabular} & $\begin{array}{c}\text { vol. } \\
\% \\
14.2 \\
15.0 \\
12.3 \\
15.3 \\
13.6\end{array}$ & $\begin{array}{c}\text { vol. } \\
\% \\
14.8 \\
15.5 \\
12.5 \\
16.1 \\
14.6\end{array}$ & $\begin{array}{l}\dot{\%} \\
95.7 \\
97.0 \\
98.9 \\
95.0 \\
93\end{array}$ & $\begin{array}{l}\text { hrs. } \\
1.5 \\
2 \\
2 \\
2.5 \\
2.5\end{array}$ \\
\hline Average & & & 34.5 & & & 96.0 & \\
\hline
\end{tabular}

hemoglobin would be greater in a normal than in a patient with polycythemia (9). In the series of cases studied by us, patients with hematocrits as high as $81 \%$ had normal oxygen saturation. Increased oxygen capacity with normal oxygen saturation was reported by Altschule and his coworkers although no figures were shown (10). Moschowitz has used arterial oxygen saturation as the important diagnostic criterion in differentiating between uncomplicated polycythemia vera and secondary erythrocytosis due to hypertension of the pulmonary circulation or prolonged mountain sickness, saturation being normal in true polycythemia (11). Hitzenberger in reporting normal arterial oxygen saturation in six patients with polycythemia vera also stresses this point (6).

It has been suggested by Bansi and Groscurth that the hemoglobin in polycythemia vera does not give up its oxygen to the tissues as readily as does normal blood (12). Isaacs, however, has shown that in polycythemia vera the hemoglobin is normal in all respects, and the oxygen unsaturation of venous blood is within normal limits (13). He found the venous unsaturation to be about 5 vol. $\%$, the same as the average found by Harrop in 15 normal cases (8). Altschule and his coworkers reported similar results $(10)$.

Fitz interpreted normal arterial oxygen saturation in erythremia as due to increased pulmonary ventilation (14). Harrop and Heath studied alveolar gas diffusion in seven cases by determining the diffusion constant for carbon monoxide, and while finding the blood oxygen content to be high and the saturation low but within normal limits, reported a decrease in the pulmonary permeability to oxygen (3). They measured blood oxygen saturation in three of the patients immediately after exercise, and found significantly lower figures than they had obtained at rest. The failure to maintain normal saturation during a time of increased metabolic demand for oxygen, they felt, was linked to poor pulmonary permeability.

Oxygen saturation of hemoglobin is a function of the partial pressure of oxygen dissolved in the blood. This pressure of oxygen is dependent on the diffusion of oxygen across the alveolus-blood barrier and is in equilibrium with the alveolar oxygen tension of about $100 \mathrm{~mm}$. $\mathrm{Hg}$. Normal oxygen saturation in polycythemia vera would thus seem to signify a normal pressure of oxygen and would further seem to indicate normal diffusion across the alveolar membrane. This is supported by additional experimental evidence to be reported.

It will be noted that the figures for oxygen saturation we report as normal are lower by a small percent than those of some other recent authors. Comroe, for example, in arterial oxygen tension studies reports an average pressure of oxygen of $93 \mathrm{~mm}$. $\mathrm{Hg}$ corresponding to about $98 \%$ oxygen saturation (15). Direct spectrophotometric studies of the circulating blood of normal dogs by Drabkin and Schmidt have given saturation values of

TABLE $V$

Results of oxygen saturation in secondary polycythemia

\begin{tabular}{|c|c|c|c|c|c|c|c|c|c|c|c|}
\hline \multirow{2}{*}{ Case No. } & \multirow{2}{*}{ Hgb. } & \multirow{2}{*}{$\underset{10 \% / \mathrm{mm}^{2}}{\stackrel{R B C}{X}}$} & \multirow{2}{*}{$\underset{\text { crit }}{\text { Hemato- }}$} & \multicolumn{4}{|c|}{ Ear } & \multicolumn{4}{|c|}{ Artery } \\
\hline & & & & $\begin{array}{c}\mathrm{O}_{2} \\
\text { Content }\end{array}$ & $\begin{array}{c}\mathrm{O}_{2} \\
\text { Capacity }\end{array}$ & $\underset{\text { Saturation }}{\mathrm{O}_{2}}$ & Time & $\begin{array}{c}\mathrm{O}_{2} \\
\text { Content }\end{array}$ & $\begin{array}{c}\mathrm{O}_{2} \\
\text { Capacity }\end{array}$ & $\mathrm{O}_{\text {Saturation }}^{\mathrm{O}_{2}}$ & Time \\
\hline $\begin{array}{l}1 \\
2 \\
3\end{array}$ & $\begin{array}{l}\text { gms. } \\
17.0 \\
17.4 \\
18.5\end{array}$ & $\begin{array}{l}6.58 \\
7.5 \\
8.1\end{array}$ & $\begin{array}{l}\% \\
58.0 \\
63 \\
65\end{array}$ & $\begin{array}{c}\text { vol. \% } \\
19.5 \\
17.3 \\
18.6\end{array}$ & $\begin{array}{c}\text { sol. \% } \\
22.1 \\
23.0 \\
24.5\end{array}$ & $\begin{array}{c}\% \\
88.0 \\
75.0 \\
76.0\end{array}$ & $\begin{array}{l}\text { hrs. } \\
3 \\
1.5 \\
3\end{array}$ & $\begin{array}{l}\text { vol. \% } \\
17.6\end{array}$ & $\begin{array}{l}\text { sol. } \% \\
23.0\end{array}$ & $\begin{array}{c}\% \\
75.2\end{array}$ & $\begin{array}{c}\text { hrs. } \\
2\end{array}$ \\
\hline Average & & & 62 & & & 79.0 & & & . & 75.2 & \\
\hline
\end{tabular}


TABLE VI

Results in 12 cases in which low arterial saturations were found

\begin{tabular}{|c|c|c|c|c|c|c|c|c|c|c|c|c|c|}
\hline \multirow{2}{*}{ Case No. } & \multirow[b]{2}{*}{ Hgb. } & \multirow{2}{*}{$\begin{array}{c}\text { RBC } \\
106 / \mathrm{mm}^{3}\end{array}$} & \multirow{2}{*}{$\begin{array}{c}\text { Hemato- } \\
\text { crit }\end{array}$} & \multirow{2}{*}{\multicolumn{2}{|c|}{ Blood Volume }} & \multicolumn{4}{|c|}{ Ear } & \multicolumn{4}{|c|}{ Artery } \\
\hline & & & & & & $\begin{array}{c}\mathrm{O}_{2} \\
\text { Content }\end{array}$ & $\begin{array}{c}\mathrm{O}_{2} \\
\text { Capacity }\end{array}$ & $\begin{array}{l}\mathrm{O}_{2} \\
\text { Satu- } \\
\text { ration }\end{array}$ & Time & $\begin{array}{l}\mathrm{O}_{2} \\
\text { Content }\end{array}$ & $\mathrm{O}_{2} \mathrm{O}_{2}$ & $\begin{array}{c}\mathrm{O}_{2} \\
\text { Satu- } \\
\text { ration }\end{array}$ & Time \\
\hline 1 & $\begin{array}{c}g m s . \\
14.2\end{array}$ & 8.25 & $\begin{array}{r}\% \\
48\end{array}$ & $\begin{array}{l}\text { liters } \\
R B C \\
2.9\end{array}$ & $\begin{array}{c}\text { liters } \\
\text { plasma } \\
3.1\end{array}$ & $\begin{array}{c}\text { vol. \% } \\
16.8\end{array}$ & $\begin{array}{c}\text { vol. \% } \\
18.9\end{array}$ & $\begin{array}{c}\% \\
88.8\end{array}$ & $\begin{array}{r}\text { hrs. } \\
18\end{array}$ & $\begin{array}{c}\text { vol. \% } \\
16.8\end{array}$ & $\begin{array}{c}\text { vol. \% } \\
18.9\end{array}$ & $\begin{array}{c}\% \\
88.8\end{array}$ & $\begin{array}{r}\text { hrs. } \\
18\end{array}$ \\
\hline 2 & 15.95 & 6.74 & 55 & & & 20.3 & 21.2 & 96.1 & 3 & & & & \\
\hline 3 & $\begin{array}{l}15.4 \\
15.8\end{array}$ & $\begin{array}{l}4.78 \\
5.4\end{array}$ & $\begin{array}{l}44 \\
46\end{array}$ & & & $\begin{array}{l}19.6 \\
18\end{array}$ & $\begin{array}{l}20.5 \\
21.1\end{array}$ & $\begin{array}{l}95.5 \\
85.4\end{array}$ & $\begin{array}{l}2 \\
8\end{array}$ & & & & \\
\hline 4 & $\begin{array}{l}14.35 \\
18.5\end{array}$ & $\begin{array}{l}5.39 \\
7.51\end{array}$ & $\begin{array}{l}46.4 \\
55\end{array}$ & & & $\begin{array}{l}16 \\
22.6\end{array}$ & $\begin{array}{l}18.9 \\
24.5\end{array}$ & $\begin{array}{l}85 \\
92\end{array}$ & $\begin{array}{l}6 \\
4\end{array}$ & & & & \\
\hline 5 & $\begin{array}{l}19.3 \\
14.9\end{array}$ & $\begin{array}{l}7.89 \\
5.88\end{array}$ & $\begin{array}{l}57 \\
47\end{array}$ & & & $\begin{array}{l}23.3 \\
17.7\end{array}$ & $\begin{array}{l}25.7 \\
19.8\end{array}$ & $\begin{array}{l}90.5 \\
89.8\end{array}$ & $\begin{array}{l}5 \\
7\end{array}$ & & & & \\
\hline 6 & $\begin{array}{l}17.1 \\
15.0\end{array}$ & $\begin{array}{l}6.2 \\
4.57\end{array}$ & $\begin{array}{l}47 \\
42\end{array}$ & & & $\begin{array}{l}19.3 \\
17.7\end{array}$ & $\begin{array}{l}22.7 \\
19.9\end{array}$ & $\begin{array}{l}85.4 \\
88.4\end{array}$ & $\begin{array}{r}8 \\
10\end{array}$ & $\begin{array}{l}20.0 \\
17.2\end{array}$ & $\begin{array}{l}22.8 \\
19.9\end{array}$ & $\begin{array}{l}88 \\
86.5\end{array}$ & $\begin{array}{l}7 \\
9\end{array}$ \\
\hline $\begin{array}{r}7 \\
8 \\
9 \\
10 \\
11 \\
12\end{array}$ & $\begin{array}{l}14.0 \\
15.0 \\
14.5 \\
21.0 \\
18.9 \\
15.95\end{array}$ & $\begin{array}{l}5.2 \\
4.53 \\
7.56 \\
7.35 \\
7.9 \\
5.75\end{array}$ & $\begin{array}{l}45 \\
44 \\
46.1 \\
65 \\
60 \\
56\end{array}$ & & & $\begin{array}{l}16.0 \\
16.8 \\
16.2 \\
25 \\
21.7 \\
18.3\end{array}$ & $\begin{array}{l}19.1 \\
19.9 \\
19.3 \\
27.9 \\
25.2 \\
21.0\end{array}$ & $\begin{array}{l}84 \\
84.4 \\
84.5 \\
89.4 \\
86 \\
87\end{array}$ & $\begin{array}{r}7 \\
6 \\
11 \\
9 \\
6 \\
7\end{array}$ & $\begin{array}{l}16.7 \\
16.2 \\
24.4\end{array}$ & $\begin{array}{l}20.0 \\
19.4 \\
28\end{array}$ & $\begin{array}{l}83.5 \\
83.5 \\
87\end{array}$ & $\begin{array}{r}6 \\
10 \\
8\end{array}$ \\
\hline Average & & & & & & & & 86.2 & & & & 83.6 & \\
\hline
\end{tabular}

98.5\% ; similar determinations carried out on normal human subjects gave figures as high as $98 \%$ to $99.3 \%(16,17)$. They concluded that determinations performed by the usual gasometric techniques are too low by about $2 \%$ or more.

Ammundsen has shown that normal blood may contain "inactive hemoglobin" equivalent to as much as 2.5 vol.\% CO capacity (18). In analyzing the errors of the gasometric method Roughton, Darling, and Root conclude that traces of $\mathrm{COHb}$, which dissociate slightly during oxygenation to increase the effective oxygen capacity, and the conversion to normal hemoglobin of some of the "inactive hemoglobin" described by Ammundsen together with other minor factors give readings which are too low by about $2 \%$ (19).

In 12 cases studied at the onset of this investigation, a total of 16 oxygen saturation determinations from ear blood and six from the radial artery were made and the results were very low with an average of $86.2 \%$ (Table VI). Only two of the ear determinations were within the normal limits (95.5\% and $96.1 \% \mathrm{O}_{2}$ saturation); the remaining 14 results ranged from $84 \%$ to $92 \%$. The arterial oxygen saturation results in six of these cases done at the same time ranged from $83.5 \%$ to $88 \%$ with a mean of $83.6 \%$. Subsequent determinations in four of these cases revealed normal oxygen saturation results. It was later demonstrated that these low results were due to the fact that the blood was permitted to stand for hours before the oxygen capacity was determined. Correcting this error in technique increased the values and brought the oxygen saturation determinations closer to normal. In polycythemia vera there is an increase in total circulating red cell mass and thus probably an increase in carboxyhemoglobin and in inactive hemoglobin. Unless the $\mathrm{O}_{2}$ content and capacity determinations are done shortly after withdrawal of the blood, there may be a release of the formerly unavailable hemoglobin with a consequent increase in the oxygen capacity leading to an erroneously low value for the oxygen saturation.

It seems apparent that in the resting patient with polycythemia vera, there is no great deficiency in the saturation of the circulating hemoglobin with oxygen. The values for the oxygen saturation in all the conditions studied except secondary erythrocytosis probably fall within normal limits. 
There is an increase in oxygen content and capacity which is proportional to the increased circulating red cell mass. Where the hematocrit is normal, the oxygen content and capacity are similarly normal and the oxygen saturation is about 95\%. There of course remains the possibility that during exercise there is significant unsaturation as suggested by Harrop and Heath (3) and this question is now under investigation in this laboratory.

\section{SUM MARY}

(1). Arterial blood oxygen saturation in polycythemia vera is within the limits of normal in the resting subject. (2). Low oxygen saturation results may be due to many factors amongst which are the inherent errors of any gasometric technique as well as the presence of traces of carboxyhemoglobin and inactive hemoglobin. (3). Gasometric oxygen determinations must be done soon after the blood is taken to prevent erroneously low oxygen saturation results.

\section{BIBLIOGRAPHY}

1. Gofman, J., Jones, H., Kelly, L., and Dobson, E., To be published.

2. Roughton, F. J. W., and Scholander, P. F., Micro gasometric estimation of the blood gases. J. Biol. Chem., 1943, 148, 541.

3. Harrop, G. A., Jr., and Heath, E. H., Pulmonary gas diffusion in polycythemia vera. J. Clin. Invest., 1927, 4, 53.

4. Barach, A. L., and McAlpin, K. R., Negative results of oxygen therapy in polycythemia vera. Am. J. M. Sc., 1933, 185, 178.

5. Brooks, W. D. W., Circulatory adjustments in polycythemia rubra vera. Proc. Roy. Soc. Med., 1936, $29,1379$.

6. Hitzenberger, K., Oxygen saturation of arterial blood in cases of polycythemia. Ztschr. f. klin. Med., 1934, 126, 495.
7. Stewart, H. J., Wheeler, C. H., and Crane, N. F., Circulatory adjustments in polycythemia vera. Am. Heart J., 1941, 21, 511.

8. Harrop, G. A., Jr., Oxygen and carbon dioxide content of arterial and venous blood in normal individuals and in patients with anemia and heart disease. J. Exper. Med., 1919, 30, 241.

9. Bauer, E., Lawrowsky, K., and Skujin, E., ther die Korrelation zwischen Sauerstoffkapazität und Gesamtmenge des hämoglobins. Ztschr. f. d. ges. exper. Med., 1927, 58, 586.

10. Altschule, M. D., Volk, M. C., and Henstell, H., Cardiac and respiratory function at rest in patients with uncomplicated polycythemia vera. Am. J. M. Sc., 1940, 200, 478.

11. Moschowitz, Eli, Essays on biology of disease; the biology of polycythemia vera. J. Mt. Sinai Hosp., 1944, 11, 232.

12. Bansi, H. W., and Groscurth, G., Veränderungen der Sauerstoffbindungskurven des Blutes bei Stoffwechsel und Blutkrankheiten (Anämie und Polycythämie). Ztschr. f. klin. Med., 1930, 113, 560.

13. Isaacs, R., Pathologic physiology of polycythemia vera. Arch. Int. Med., 1923, 31, 289.

14. Fitz, F., Erythremia in: Oxford Medicine, Christian, H. A., Oxford Press, New York, Vol. 2, p. 763.

15. Comroe, J. H., Normal human arterial oxygen tension. Am. J. Med. Sc., 1944, 208, 135.

16. Drabkin, D. L., and Schmidt, C. F., Spectrophotometric observation of circulating blood in vivo, and the direct spectrophotometric determination of the saturation of hemoglobin in arterial dog blood. Am. J. Med. Sc., 1944, 208, 133.

17. Drabkin, D. L., Schmidt, C. F., Bruner, H. D., and Pennes, H. H., The direct spectrophotometric determination of the saturation of hemoglobin in the arterial blood of man. Am. J. M. Sc., 1944, 208, 135.

18. Ammundsen, E., Studies on presence of non-carbon monoxide-combining (inactive) hemoglobin in blood of normal persons. J. Biol. Chem., 1941, 138, 563.

19. Roughton, F. J. W., Darling, R. C., and Root, W. S., Factors affecting the determination of oxygen capacity, content, and pressure in human arterial blood. Am. J. M. Sc., 1944, 208, 132. 